
involving a weighted sequential fractional derivative

Khaled M. Furati 



\title{
Bounds on the solution of a Cauchy-type problem involving a weighted sequential fractional derivative
}

\author{
Khaled M. Furati * \\ Department of Mathematics \& Statistics \\ King Fahd University of Petroleum \& Minerals \\ Dhahran 31261, Saudi Arabia
}

\begin{abstract}
In this paper we establish some bounds for the solution of a Cauchy-type problem for a class of fractional differential equations with a weighted sequential fractional derivative. The bounds are based on a Bihari-type inequality and a bound on Gauss hypergeometric function.
\end{abstract}

Keywords: fractional derivatives; Riemann-Liouville fractional derivative; Caputo fractional derivative; fractional differential equation; generalized Riemann-Liouville fractional derivative.

2010 Mathematics Subject Classification: 26A33, 34A08, 34A34, 34A12, 45J05

\section{Introduction}

Fractional calculus approach has been increasingly introduced and adopted in many models. It has been proven that fractional models provide a tool for capturing and understanding complex phenomena in many areas. Indeed, some of these models are supported by experimental evidence and yield results that agree with the observed behavior. See for example the surveys in $[1,2]$ and the collection of applications in [3].

Some recent applications include control systems [4,5], viscoelasticity [6-9], and nanotechnology [10]. Also fractional models are used to model a vibrating string [11], anomalous transport [12], and anomalous diffusion [13-16].

Another field of applications is random walk and stochastic processes [17-19] and their applications in financial modeling [20-22]. Other physical and engineering processes are given in $[23,24]$

As a result, there has been a considerable interest in the theory of fractional differential equations. The investigations include the existence and uniqueness of solutions, asymptotic behavior, stability, etc. See for example the books $[1,25,26]$ and the articles [27-33], and the references therein.

In this paper we consider a Cauchy-type problem associated with the equation

$$
\mathcal{D}_{r, a}^{\alpha, \beta} u(t)=f(t, u(t)), \quad a<t \leq b, \quad 0 \leq r<\alpha<1, \quad 0<\beta<1,
$$

\footnotetext{
${ }^{*}$ kmfurati@kfupm.edu.sa
} 
where $\mathcal{D}_{r, a}^{\alpha, \beta}$ is the weighted sequential fractional derivative introduced in [34].

In a series of articles, [35-37], Glushak studied the uniform well-posedness of a Cauchytype problem with two fractional derivatives and bounded operator. He also proposed a criterion for the uniform correctness of unbounded operator. In [34,38] an existence and uniqueness result is obtained.

Next, we start with some preliminaries in Section 2. In Section 3 we define the sequential integrals and derivatives and present some properties. In Section 4 we construct some bounds on the sequential fractional integral. In Section 5 we obtain a bound on the solution of the Cauchy problem.

\section{Preliminaries}

In this section we present some definitions, lemmas, properties and notation which we use later. For more details please see [1].

Let $-\infty<a<b<\infty$. Let $C[a, b]$ and $C(a, b)$ denote the space of continuous functions on $[a, b]$ and $(a, b)$, respectively. We denote by $L^{p}(a, b), p \geq 1$, the spaces of Lebesgue integrable functions on $(a, b)$, in particular we use $L(a, b)$ to denote $L^{1}(a, b)$. We denote the subspace $C(a, b) \cap L(a, b)$ by $C L(a, b)$. Furthermore, we introduce the following weighted spaces of continuous functions

$$
C_{\omega}[a, b]=\left\{f:(a, b] \rightarrow \mathbb{R}:(t-a)^{\omega} f(t) \in C[a, b]\right\}, \quad 0 \leq \omega<1,
$$

with the norm

$$
\|f\|_{C_{\omega}}=\left\|(t-a)^{\omega} f(t)\right\|_{C} .
$$

The following is a special case of Jensen's Inequality.

Lemma 1 For nonnegative $a_{i}, i=1, \ldots, k$,

$$
\left(\sum_{i=1}^{k} a_{i}\right)^{p} \leq k^{p-1} \sum_{i=1}^{k} a_{i}^{p}, \quad p \geq 1 .
$$

The following lemma is given in [39], theorem 2.5.1.

Lemma 2 Let $0<T \leq \infty$. Let $a(t)$ and $b(t)$ be continuous positive functions defined on $[0, T)$. Let $w:[0, \infty) \rightarrow[0, \infty)$ be a continuous monotonic nondecreasing function such that $w(0)=0$ and $w(t)>0$ for $t>0$. If $u$ is a positive differentiable function on $[0, T)$ that satisfies

$$
u^{\prime}(t) \leq a(t) w(u(t))+b(t), \quad t \in[0, T),
$$

then we have

$$
u(t) \leq G^{-1}\left[G\left(u(0)+\int_{0}^{t} b(s) d s\right)+\int_{0}^{t} a(s) d s\right],
$$

for the values of $t \in[0, T)$ for which the right-hand side is well-defined, where

$$
G(r)=\int_{r_{0}}^{r} \frac{d s}{w(s)}, \quad r>0, r_{0}>0 .
$$

The next lemma is a special case of Lemma 2. 
Lemma 3 Let $a(t)$ and $b(t)$ be continuous positive functions defined on $[0, T), T>0$. Let $u$ be a positive differentiable function on $[0, T)$ that satisfies

$$
u^{\prime}(t) \leq a(t) u^{m}(t)+b(t), \quad t \in[0, T), \quad m>0 .
$$

Let

$$
A(t)=\int_{0}^{t} a(s) d s, \quad B(t)=u(0)+\int_{0}^{t} b(s) d s .
$$

Then for $m>0, m \neq 1$, we have

$$
u(t) \leq\left[(1-m) A(t)+B^{1-m}(t)\right]^{\frac{1}{1-m}},
$$

for $t \in\left[0, T_{m}\right)$ where

$$
T_{m}= \begin{cases}T, & 0<m<1, \\ \max \left\{t \in[0, T): A(t) B^{m-1}(t)<\frac{1}{m-1}\right\}, & m>1 .\end{cases}
$$

For $m=1$ we have

$$
u(t) \leq e^{A(t)} B(t),
$$

for $t \in[0, T)$.

Proof. Applying lemma 2 with $w(u)=u^{m}$,

$$
G(r)=\int_{r_{0}}^{r} \frac{d s}{s^{m}}= \begin{cases}\frac{1}{1-m}\left(r^{1-m}-r_{0}^{1-m}\right), & m>0, m \neq 1, \\ \ln r, & m=1 .\end{cases}
$$

and

$$
G^{-1}(y)= \begin{cases}{\left[r_{0}^{1-m}-(m-1) y\right]^{\frac{1}{1-m}},} & m>0, m \neq 1, \\ e^{y}, & m=1 .\end{cases}
$$

Thus for $m>0, m \neq 1$, the right-hand side of (3) takes the form

$$
\begin{aligned}
& G^{-1}\left[G\left(u(0)+\int_{0}^{t} b(s) d s\right)+\int_{0}^{t} a(s) d s\right]=G^{-1}[G(B(t))+A(t)] \\
& =\left[r_{0}^{1-m}-(m-1)[G(B(t))+A(t)]\right]^{\frac{1}{1-m}} \\
& =\left[r_{0}^{1-m}-(m-1) A(t)-(m-1) \frac{1}{1-m}\left(B^{1-m}(t)-r_{0}^{1-m}\right)\right]^{\frac{1}{1-m}} \\
& =\left[r_{0}^{1-m}-(m-1) A(t)+B^{1-m}(t)-r_{0}^{1-m}\right]^{\frac{1}{1-m}} \\
& =\left[(1-m) A(t)+B^{1-m}(t)\right]^{\frac{1}{1-m}} .
\end{aligned}
$$

If $0<m<1$ then the expression inside the bracket is nonnegative and thus the power of this expression is well defined for all $t \in[0, T)$. For $m>1$, the first term in the bracket is negative for $t>0$ and thus the bracket might not be nonnegative for sufficiently large $t$ in general.

For $m=1$, the result follows by substituting the case $m=1$ of (6) and (7) in the inequality (3).

The left-sided Riemann-Liouville fractional integrals and derivatives are defined as follows. 
Definition 4 Let $f \in L(a, b)$. The integral

$$
I_{a^{+}}^{\alpha} f(t):=\frac{1}{\Gamma(\alpha)} \int_{a}^{x} \frac{f(s)}{(x-s)^{1-\alpha}} d s, \quad t>a, \quad \alpha>0,
$$

is called the left-sided Riemann-Liouville fractional integral of order $\alpha$ of the function $f$.

Definition 5 The expression

$$
D_{a^{+}}^{\alpha} f(t):=D I_{a^{+}}^{\alpha-1} f(t), \quad t>a, \quad 0<\alpha<1, \quad D=\frac{d}{d t},
$$

is called the left-sided Riemann-Liouville fractional derivative of order $\alpha$ of $f$ provided the right-hand side exists.

Lemma 6 ( [40]) Let $\lambda, \nu, \eta>0$, then

$$
I^{\nu}\left[t^{\lambda-1} e^{-\eta t}\right]:=\frac{1}{\Gamma(\nu)} \int_{0}^{t}(t-s)^{\nu-1} s^{\lambda-1} e^{-\eta s} d s \leq A t^{\nu-1}, \quad t>0,
$$

where

$$
A=\max \left\{1,2^{1-\nu}\right\} \frac{\Gamma(\lambda)}{\Gamma(\nu)}\left(1+\frac{\lambda(\lambda+1)}{\nu}\right) \eta^{-\lambda}>0 .
$$

Now we introduce some properties of the Gauss hypergeometric function ${ }_{2} F_{1}$. From formula 5, Table 9.1 in [41], we have the following lemma.

Lemma 7 Let $\alpha>0, \beta>0, r \in \mathbb{R}$, and $0<s<t$, then

$$
\int_{s}^{t}(\tau-s)^{\alpha-1}(t-\tau)^{\beta-1} \tau^{-r} d \tau=\frac{\Gamma(\alpha) \Gamma(\beta)}{\Gamma(\alpha+\beta)}(t-s)^{\alpha+\beta-1} s_{2}^{-r} F_{1}\left(\alpha, r ; \alpha+\beta ; 1-\frac{t}{s}\right) .
$$

The following bound is given in [42], chapter 4, Lemma 4.1 .

Lemma 8 Let $a, b$ and $c$ be complex numbers and let $\delta>0$. Then there is a constant $M$, independent of $\nu \in(0,1)$, such that

$$
\left|{ }_{2} F_{1}\left(a, b ; c ; 1-\frac{1}{\nu}\right)\right| \leq M \Gamma(c) \nu^{\xi}, \quad \xi=\min \{\operatorname{Re} a, \operatorname{Re} b\}-\delta .
$$

Thus from lemma 7 and lemma 8 we have

Lemma 9 Let $\alpha>0, \beta>0$, and $r \in \mathbb{R}$. Given $\delta>0$, there exists a constant $M>0$ such that for $0<s<t$,

$$
0 \leq{ }_{2} F_{1}\left(\alpha, r ; \alpha+\beta ; 1-\frac{t}{s}\right) \leq M \Gamma(\alpha+\beta)\left(\frac{s}{t}\right)^{\xi}, \quad \xi=\min \{\alpha, r\}-\delta .
$$




\section{Sequential integrals and derivatives}

Definition 10 Let $\alpha>0, \beta>0, r \in \mathbb{R}$. Let $f \in C L(a, b)$. Define the sequential integral $\mathcal{J}_{r, a}^{\alpha, \beta} f$ and the sequential derivative $\mathcal{D}_{r, a}^{\alpha, \beta} f$ by

$$
\mathcal{J}_{r, a}^{\alpha, \beta} f(t)=I_{a}^{\alpha}(x-a)^{-r} I_{a}^{\beta} f(t),
$$

and

$$
\mathcal{D}_{r, a}^{\alpha, \beta} f(t)=D_{a}^{\alpha}(x-a)^{r} D_{a}^{\beta} f(t),
$$

if the right-hand sides exist.

Without loss of generality we take $a=0$ and drop the subscript $a$.

The integral $\mathcal{J}_{r}^{\alpha, \beta} f$ can be represented in terms of the Gauss hypergeometric function ${ }_{2} F_{1}$ as follows.

Lemma 11 Let $\alpha>0, \beta>0$, and $r \in \mathbb{R}$. Let $f$ be a measurable nonnegative function defined on $(0, t)$. If $\mathcal{J}_{r}^{\beta, \alpha} f(t)$ exists then

$$
\mathcal{J}_{r}^{\beta, \alpha} f(t)=\frac{1}{\Gamma(\alpha+\beta)} \int_{0}^{t}(t-s)^{\alpha+\beta-1} s_{2}^{-r} F_{1}\left(\alpha, r ; \alpha+\beta ; 1-\frac{t}{s}\right) f(s) d s .
$$

Proof. Using Dirichlet formula, we obtain

$$
\begin{aligned}
I^{\beta}\left[t^{-r} I^{\alpha} f(t)\right] & :=\frac{1}{\Gamma(\beta)} \int_{0}^{t}(t-\tau)^{\beta-1} \tau^{-r} I^{\alpha} f(\tau) d \tau \\
& :=\frac{1}{\Gamma(\beta) \Gamma(\alpha)} \int_{0}^{t}(t-\tau)^{\beta-1} \tau^{-r}\left[\int_{0}^{\tau}(\tau-s)^{\alpha-1} f(s) d s\right] d \tau \\
& =\frac{1}{\Gamma(\beta) \Gamma(\alpha)} \int_{0}^{t} \int_{0}^{\tau}(t-\tau)^{\beta-1} \tau^{-r}(\tau-s)^{\alpha-1} f(s) d s d \tau \\
& =\frac{1}{\Gamma(\beta) \Gamma(\alpha)} \int_{0}^{t}\left[\int_{s}^{t}(\tau-s)^{\alpha-1}(t-\tau)^{\beta-1} \tau^{-r} d \tau\right] f(s) d s .
\end{aligned}
$$

Thus, formula (10) follows by substitution from lemma 7 .

Now formula (10) and lemma 9 lead to the following bound.

Lemma 12 Let $\alpha>0, \beta>0$, and $r \in \mathbb{R}$. Let $f$ be a measurable nonnegative function defined on $(0, t)$. If $\mathcal{J}_{r}^{\beta, \alpha} f(t)$ exists then given $\delta>0$, there exists a constant $M>0$ such that

$$
\mathcal{J}_{r}^{\beta, \alpha} f(t) \leq M t^{-\xi} \Gamma(\alpha+\beta) I^{\alpha+\beta}\left[t^{\xi-r} f(t)\right], \quad \xi=\min \{\alpha, r\}-\delta .
$$

Proof.

$$
\begin{aligned}
\mathcal{J}_{r}^{\beta, \alpha} f(t) & =\frac{1}{\Gamma(\alpha+\beta)} \int_{0}^{t}(t-s)^{\alpha+\beta-1} s^{-r}{ }_{2} F_{1}\left(\alpha, r ; \alpha+\beta ; 1-\frac{t}{s}\right) f(s) d s \\
& \leq \frac{1}{\Gamma(\alpha+\beta)} \int_{0}^{t}(t-s)^{\alpha+\beta-1} s^{-r} M \Gamma(\alpha+\beta)\left(\frac{s}{t}\right)^{\xi} f(s) d s \\
& =M t^{-\xi} \int_{0}^{t}(t-s)^{\alpha+\beta-1} s^{\xi-r} f(s) d s, \quad \xi-r=\min \{\alpha-r, 0\}-\delta . \\
& =M t^{-\xi} \Gamma(\alpha+\beta) I^{\alpha+\beta}\left[t^{\xi-r} f(t)\right] .
\end{aligned}
$$




\section{Bounds for the sequential integral}

Lemma 13 Let $\alpha>0$ and $0 \leq \omega<1$. Let $f(., u().) \in L(0, T), 0<T \leq \infty$, for any $u \in C_{\omega}[0, T]$ and satisfies

$$
|f(t, u(t))| \leq t^{\mu} e^{-\sigma t} \phi(t)|u(t)|^{m}, \quad t \in(0, T),
$$

with $m \in \mathbb{N}, \mu>m \omega-1, \sigma>0$, and $\phi$ is a continuous nonnegative function on $(0, T)$. Then

$$
\left|I^{\alpha} f(t, u(t))\right| \leq \frac{A^{1 / p}}{\Gamma(\alpha)} t^{\alpha-1}\left(\int_{0}^{t} \phi^{q}(s) s^{\omega q m}|u(s)|^{q m} d s\right)^{1 / q}, \quad t \in(0, T),
$$

for any

$$
q>\max \left\{\frac{1}{\mu-\omega m+1}, \frac{1}{\alpha}, 1\right\}
$$

where $A>0$ is given by (8) with

$$
\nu=p(\alpha-1)+1, \quad \lambda=p(\mu-\omega m)+1, \quad \eta=p \sigma,
$$

and $p$ is the conjugate of $q$, i.e. $\frac{1}{p}+\frac{1}{q}=1$.

Proof. Let $q$ be as in (14) and $p$ its conjugate. Then by Hölder inequality we obtain

$$
\begin{aligned}
& \Gamma(\alpha)\left|I^{\alpha} f(t, u(t))\right| \leq \int_{0}^{t}(t-s)^{\alpha-1}|f(s, u(s))| d s \\
& \leq \int_{0}^{t}(t-s)^{\alpha-1} s^{\mu} e^{-\sigma s} \phi(s)|u(s)|^{m} d s \\
& \leq \int_{0}^{t}(t-s)^{\alpha-1} s^{\mu-\omega m} e^{-\sigma s} \phi(s) s^{\omega m}|u(s)|^{m} d s \\
& \leq\left(\int_{0}^{t}(t-s)^{p(\alpha-1)} s^{p(\mu-\omega m)} e^{-p \sigma s} d s\right)^{1 / p}\left(\int_{0}^{t} \phi^{q}(s) s^{\omega q m}|u(s)|^{q m} d s\right)^{1 / q} .
\end{aligned}
$$

From (14) we have

$$
p(\alpha-1)=\frac{q}{q-1}(\alpha-1)=\frac{q \alpha-q}{q-1}>\frac{1-q}{q-1}=-1,
$$

and

$$
p(\mu-\omega m)=\frac{q(\mu-\omega m)}{q-1}>\frac{1-q}{q-1}=-1 .
$$

Thus we can apply lemma 6 with

$$
\nu=p(\alpha-1)+1>0, \quad \lambda=p(\mu-\omega m)+1>0, \quad \eta=p \sigma>0,
$$

to obtain

$$
\int_{0}^{t}(t-s)^{p(\alpha-1)} s^{p(\mu-\omega m)} e^{-p \sigma s} d s \leq A t^{p(\alpha-1)}
$$

where $A$ is given by (8) with $\nu, \lambda$ and $\eta$ as in (15). Finally, the inequality (13) follows by substituting (17) into (16). 
Remark 14 As for the second integral in (16), note that from the hypothesis we have $t^{\omega q m}|u|^{q m} \in C[0, T]$. So if $\phi \in L^{q}(0, T)$ then the integral is finite.

Lemma 15 Let $\alpha>0, \beta>0, r \in \mathbb{R}$, and $0 \leq \omega<1$. Let $f(., u().) \in L(0, T)$, $0<T \leq \infty$, for any $u \in C_{\omega}[0, T]$ and satisfies

$$
|f(t, u(t))| \leq t^{\mu} e^{-\sigma t} \phi(t)|u(t)|^{m}, \quad t \in(0, T),
$$

with $m \in \mathbb{N}, \mu>\omega m-1-\min \{\alpha-r, 0\}, \sigma>0$, and $\phi$ is a continuous nonnegative function on $(0, T)$.

If $\mathcal{J}_{r}^{\beta, \alpha} f(t, u(t))$ exists then given

$$
0<\delta<\mu-\omega m+1+\min \{\alpha-r, 0\}
$$

there exists $M>0$ such that

$$
\left|\mathcal{J}_{r}^{\beta, \alpha} f(t, u(t))\right| \leq A^{1 / p} M t^{\alpha+\beta-\xi-1}\left(\int_{0}^{t} \phi^{q}(s) s^{q m w}|u(s)|^{q m} d s\right)^{1 / q},
$$

for any

$$
q>\max \left\{\frac{1}{\mu+\xi-r-\omega m+1}, \frac{1}{\alpha+\beta}, 1\right\},
$$

where $A>0$ is given by (8) with

$$
\nu=p(\alpha+\beta-1)+1, \quad \lambda=p(\mu+\xi-r-\omega m)+1, \quad \eta=p \sigma,
$$

$p$ is the conjugate of $q$, and $\xi=\min \{\alpha, r\}-\delta$.

Proof. From lemma 12, given $0<\delta<\mu-\omega m+1+\min \{\alpha-r, 0\}$, there exists $M>0$ such that

$$
\left|\mathcal{J}_{r}^{\beta, \alpha} f(t, u(t))\right| \leq \mathcal{J}_{r}^{\beta, \alpha}|f(t, u(t))| \leq M t^{-\xi} \Gamma(\alpha+\beta) I^{\alpha+\beta}\left|t^{\xi-r} f(t, u(t))\right|,
$$

where $\xi=\min \{\alpha, r\}-\delta$. Moreover, for any $\delta$ that satisfies (19) we have

$$
\mu+\xi-r-\omega m+1>\delta-\min \{\alpha-r, 0\}+\xi-r=0,
$$

since $\xi-r=\min \{\alpha-r, 0\}-\delta$. Also from hypothesis (18),

$$
t^{\xi-r}|f(t, u(t))| \leq t^{\mu+\xi-r} e^{-\sigma t} \phi(t)|u(t)|^{m} .
$$

Thus we can apply Lemma 13 with $\alpha$ and $\mu$ replaced by $\alpha+\beta$ and $\mu+\xi-r$, respectively, to obtain the bound

$$
I^{\alpha+\beta}\left|t^{\xi-r} f(t, u(t))\right| \leq \frac{A^{1 / p}}{\Gamma(\alpha+\beta)} t^{\alpha+\beta-1}\left(\int_{0}^{t} \phi^{q}(s) s^{q m w}|u(s)|^{q m} d s\right)^{1 / q},
$$

where $A$ is given by (8) with $\nu, \lambda$ and $\eta$ as in (22). Now (20) follows by combining (23) and (24).

An alternative bound can be obtained using Dirichlet formula. 
Lemma 16 Let $0 \leq r<\alpha, \beta>0$, and $0 \leq \omega<1$. Let $f(., u().) \in L(0, T), 0<T \leq \infty$, for any $u \in C_{\omega}[0, T]$ and satisfies

$$
|f(t, u(t))| \leq t^{\mu} e^{-\sigma t} \phi(t)|u(t)|^{m}, \quad t \in(0, T),
$$

with $m \in \mathbb{N}, \mu-m \omega>-1, \sigma>0$, and $\phi$ is a continuous nonnegative continuous function on $(0, T)$. Then

$$
\left|\mathcal{J}_{r}^{\beta, \alpha} f(t, u(t))\right| \leq K t^{\alpha+\beta-r-1}\left(\int_{0}^{t} \phi^{q}(s) s^{\omega q m}|u(s)|^{q m} d s\right)^{1 / q},
$$

for any

$$
q>\max \left\{\frac{1}{\mu-\omega m+1}, \frac{1}{\alpha}, 1, \frac{1}{\beta}, \frac{1}{\alpha-r}\right\}
$$

where

$$
K=\frac{A^{1 / p}}{\Gamma(\alpha) \Gamma(\beta)}\left(\frac{\Gamma(p(\beta-1)+1) \Gamma(p(\alpha-r-1)+1)}{\Gamma(p(\alpha-r+\beta-2)+2)}\right)^{1 / p}
$$

$A>0$ is given by (8) with

$$
\nu=p(\alpha-1)+1, \quad \lambda=p(\mu-\omega m)+1, \quad \eta=p \sigma,
$$

and $p$ is the conjugate of $q$.

Proof. Let $v(t)=t^{\omega}|u(t)|$, then $v \in C[0, T]$. Since the hypothesis of Lemma 13 hold, using Hölder inequality we obtain the following bound.

$$
\begin{aligned}
& \left|\mathcal{J}_{r}^{\beta, \alpha} f(t, u(t))\right| \\
& =\left|I^{\beta} t^{-r} I^{\alpha} f(t, u(t))\right| \leq I^{\beta} t^{-r}\left|I^{\alpha} f(t, u(t))\right| \\
& \leq \frac{A^{1 / p}}{\Gamma(\alpha)} I^{\beta}\left[t^{-r} t^{\alpha-1}\left(\int_{0}^{t} \phi^{q}(s) v^{q m}(s) d s\right)^{1 / q}\right] \\
& =\frac{A^{1 / p}}{\Gamma(\alpha) \Gamma(\beta)} \int_{0}^{t}(t-s)^{\beta-1} s^{\alpha-r-1}\left(\int_{0}^{s} \phi^{q}(\tau) v^{q m}(\tau) d \tau\right)^{1 / q} d s \\
& =\frac{A^{1 / p}}{\Gamma(\alpha) \Gamma(\beta)}\left(\int_{0}^{t}(t-s)^{p(\beta-1)} s^{p(\alpha-r-1)} d s\right)^{1 / p} \\
& \quad \times\left(\int_{0}^{t} \int_{0}^{s} \phi^{q}(\tau) v^{q m}(\tau) d \tau d s\right)^{1 / q} .
\end{aligned}
$$

From (27), the powers inside the first integral satisfy

$$
p(\beta-1)>p\left(\frac{1}{q}-1\right)=\frac{p}{q}-p=-1,
$$

and

$$
p(\alpha-r-1)>p\left(\frac{1}{q}-1\right)=-1 .
$$


Thus this integral is finite and given by

$$
\begin{aligned}
\int_{0}^{t}(t-s)^{p(\beta-1)} s^{p(\alpha-r-1)} d s & =\Gamma(p(\beta-1)+1) I^{p(\beta-1)+1} t^{p(\alpha-r-1)} \\
& =\frac{\Gamma(p(\beta-1)+1) \Gamma(p(\alpha-r-1)+1)}{\Gamma(p(\alpha-r-1)+p(\beta-1)+2)} t^{p(\alpha-r-1)+p(\beta-1)+1} \\
& =\frac{\Gamma(p(\beta-1)+1) \Gamma(p(\alpha-r-1)+1)}{\Gamma(p(\alpha-r+\beta-2)+2)} t^{p(\alpha-r+\beta-2)+1} .(31)
\end{aligned}
$$

Regarding the second integral, if it is infinite then (26) is true. If the integral is finite then using Dirichlet formula we obtain

$$
\begin{aligned}
\int_{0}^{t} \int_{0}^{s} \phi^{q}(\tau) v^{q m}(\tau) d \tau d s & =\int_{0}^{t} \int_{\tau}^{t} \phi^{q}(\tau) v^{q m}(\tau) d s d \tau \\
& =\int_{0}^{t}(t-\tau) \phi^{q}(\tau) v^{q m}(\tau) d \tau \\
& \leq t \int_{0}^{t} \phi^{q}(\tau) v^{q m}(\tau) d \tau
\end{aligned}
$$

Substituting (31) and (32) into (30) yields

$$
\begin{aligned}
&\left|\mathcal{J}_{r}^{\beta, \alpha} f(t, u(t))\right| \leq \frac{A^{1 / p}}{\Gamma(\alpha) \Gamma(\beta)}\left(\frac{\Gamma(p(\beta-1)+1) \Gamma(p(\alpha-r-1)+1)}{\Gamma(p(\alpha-r+\beta-2)+2)}\right)^{1 / p} \times \\
&= \frac{A^{\alpha-r+\beta-2+1 / p+1 / q}\left(\int_{0}^{t} \phi^{q}(\tau) v^{q m}(\tau) d \tau\right)^{1 / q}}{\Gamma(\alpha) \Gamma(\beta)}\left(\frac{\Gamma(p(\beta-1)+1) \Gamma(p(\alpha-r-1)+1)}{\Gamma(p(\alpha-r+\beta-2)+2)}\right)^{1 / p} \times \\
& t^{\alpha+\beta-r-1}\left(\int_{0}^{t} \phi^{q}(\tau) v^{q m}(\tau) d \tau\right)^{1 / q} .
\end{aligned}
$$

This completes the proof.

\section{Bounds for the solution of the Cauchy problem}

In this section we obtain a bound on the solution of the Cauchy-type problem

$$
\begin{aligned}
& \mathcal{D}_{r}^{\alpha, \beta} u(t)=f(t, u(t)), \quad t>0, \quad 0 \leq r<\alpha<1, \quad 0<\beta<1, \\
& \lim _{t \rightarrow 0^{+}} I^{1-\beta} u(t)=c_{0}, \\
& \lim _{t \rightarrow 0^{+}} I^{1-\alpha}\left[t^{r} D^{\beta} u\right](t)=c_{1},
\end{aligned}
$$

where $c_{0}$ and $c_{1}$ are real numbers.

The proof of the following lemma is similar to the proof of Theorem 27 in [34].

Lemma 17 Let $0 \leq r<\alpha<1,0<\beta<1$, and $0 \leq \omega<1$. Let $f:(0, T] \times \mathbb{R} \rightarrow \mathbb{R}$ be such that $f(., u().) \in C_{\omega}[0, T)$ for any $u \in C_{\omega}[0, T)$. 
If $u \in C_{\omega}[0, T)$ with $D^{\beta} u \in C(0, T)$ satisfies the Cauchy-type problem (33), then $u$ satisfies the integral equation,

$$
u(t)=\frac{c_{0}}{\Gamma(\beta)} t^{\beta-1}+\frac{c_{1} \Gamma(\alpha-r)}{\Gamma(\alpha) \Gamma(\alpha+\beta-r)} t^{\alpha+\beta-r-1}+\mathcal{J}_{r}^{\beta, \alpha} f(t, u(t)),
$$

for $0<t<T$.

The integral form (34) leads to the following result.

Theorem 18 Let $0 \leq r<\alpha<1,0 \leq \beta \leq 1$, and $0 \leq \omega<1$. Let $f(., u().) \in C_{\omega}[0, T)$, $0<T \leq \infty$, for any $u \in C_{\omega}[0, T)$ and satisfies

$$
|f(t, u(t))| \leq t^{\mu} e^{-\sigma t} \phi(t)|u|^{m}, \quad \mu \geq 0, \quad m \in \mathbb{N}, \quad \sigma>0,
$$

with $m \in \mathbb{N}, \mu>m \omega-1$, and $\sigma>0$. Here $\phi$ is a nonnegative function in $C_{\eta}[0, T]$ such that $\phi^{q}$ is integrable at 0 where

$$
q>\max \left\{\frac{1}{\mu-\omega m+1}, \frac{1}{\beta}, \frac{1}{\alpha-r}\right\},
$$

and

$$
\eta=m(\beta-1+\omega) .
$$

If $u \in C_{\omega}[0, T)$ with $D^{\beta} u \in C(0, T)$ is a solution of (33) then on $(0, T)$, u satisfies the inequality

$$
|u(t)| \leq\left\{\begin{array}{rr}
K t^{\alpha+\beta-r-1}\left[(1-m) A(t)+B^{1-m}(t)\right]^{\frac{1}{q(1-m)}}, & m>0, m \neq 1, \\
\frac{c_{0}}{\Gamma(\beta)} t^{\beta-1}+\frac{c_{1} \Gamma(\alpha-r)}{\Gamma(\alpha) \Gamma(\alpha+\beta-r)} t^{\alpha+\beta-r-1}+K t^{\alpha+\beta-r-1} B^{1 / q}(t) e^{A(t) / q}, \\
m=1,
\end{array}\right.
$$

where $K$ is given by (28),

$$
\begin{gathered}
A(t)=A_{2} \int_{0}^{t} s^{q m(\alpha-r)} s^{q \eta} \phi^{q}(s) d s, \\
B(t)=A_{0} \int_{0}^{t} s^{q \eta} \phi^{q}(s) d s+\frac{A_{1}}{A_{2}} A(t),
\end{gathered}
$$

and

$$
A_{0}=3^{q-1}\left(\frac{c_{0}}{\Gamma(\beta)}\right)^{q m}, \quad A_{1}=3^{q-1}\left(\frac{c_{1} \Gamma(\alpha-r)}{\Gamma(\alpha) \Gamma(\alpha+\beta-r)}\right)^{q m}, \quad A_{2}=3^{q-1} K^{q m} .
$$

Proof. Since the hypothesis of Lemma 17 are satisfied, the solution $u$ of the problem 33 satisfies the nonlinear integral equation (34). From the bound (26) in Lemma 16 we obtain the inequality

$$
\begin{aligned}
|u(t)| \leq \frac{c_{0}}{\Gamma(\beta)} & t^{\beta-1}+\frac{c_{1} \Gamma(\alpha-r)}{\Gamma(\alpha) \Gamma(\alpha+\beta-r)} t^{\alpha+\beta-r-1} \\
& +K t^{\alpha+\beta-r-1}\left(\int_{0}^{t} \phi^{q}(s)\left|s^{w} u(s)\right|^{q m} d s\right)^{1 / q} .
\end{aligned}
$$


Multiplying both sides of (42) by $t^{w}$ we can write

$$
\begin{aligned}
t^{w}|u(t)| \leq \frac{c_{0}}{\Gamma(\beta)} t^{\beta-1+\omega}+\frac{c_{1} \Gamma(\alpha-r)}{\Gamma(\alpha) \Gamma(\alpha+\beta-r)} t^{\alpha+\beta-r-1+\omega} \\
\\
+K t^{\alpha+\beta-r-1+\omega}\left(\int_{0}^{t} \phi^{q}(s)\left|s^{w} u(s)\right|^{q m} d s\right)^{1 / q} .
\end{aligned}
$$

Let $z(t)=t^{w}|u(t)|$, then

$$
z(t) \leq \frac{c_{0}}{\Gamma(\beta)} t^{\beta-1+\omega}+\frac{c_{1} \Gamma(\alpha-r)}{\Gamma(\alpha) \Gamma(\alpha+\beta-r)} t^{\alpha+\beta-r-1+\omega}+K t^{\alpha+\beta-r-1+\omega} g^{1 / q}(t),
$$

where

$$
g(t)=\int_{0}^{t} \phi^{q}(s) z^{q m}(s) d s .
$$

Note that it follows from the assumptions on $\phi$ and $u$ that $g \in A C[0, T]$. From Lemma 1 we obtain

$$
\begin{aligned}
g^{\prime}(t) & =\phi^{q}(t) z^{q m}(t) \\
& \leq \phi^{q}(t)\left[A_{0} t^{q m(\beta-1+\omega)}+A_{1} t^{q m(\alpha+\beta-r-1+\omega)}+A_{2} t^{q m(\alpha+\beta-r-1+\omega)} g^{m}(t)\right] \\
& =\phi^{q}(t)\left[A_{0} t^{q \eta}+A_{1} t^{q(\eta+m(\alpha-r))}+A_{2} t^{q(\eta+m(\alpha-r))} g^{m}(t)\right] .
\end{aligned}
$$

where $A_{0}, A_{1}$, and $A_{2}$ are given by (41). Thus we can write

$$
g^{\prime}(t) \leq a(t) g^{m}(t)+b(t),
$$

where

$$
a(t)=A_{2} t^{q(\eta+m(\alpha-r))} \phi^{q}(t)=A_{2} t^{q m(\alpha-r))}\left[t^{\eta} \phi(t)\right]^{q},
$$

and

$$
b(t)=\left[A_{0} t^{q \eta}+A_{1} t^{q(\eta+m(\alpha-r))}\right] \phi^{q}(t)=A_{0}\left[t^{\eta} \phi(t)\right]^{q}+\frac{A_{1}}{A_{2}} a(t) .
$$

Note that $a, b \in C[0, T]$. Note that $g$ is nonnegative and continuous in $(0, T)$. Furthermore, $g$ is also nondecreasing since $g^{\prime}$ is nonnegative. Thus by Lemma 3 , we have two cases.

Case 1. $m>0, m \neq 1$. Then for $t \in\left[0, T_{m}\right)$, where $T_{m}$ is as given by (5),

$$
g(t) \leq\left[(1-m) A(t)+B^{1-m}(t)\right]^{\frac{1}{1-m}},
$$

where $A(t)$ and $B(t)$ are as given in (39) and (40), respectively.

Thus

$$
\begin{aligned}
& |u(t)|=t^{-\omega} z(t) \\
& \leq t^{-\omega}\left\{\frac{c_{0}}{\Gamma(\beta)} t^{\beta-1+\omega}+\frac{c_{1} \Gamma(\alpha-r)}{\Gamma(\alpha) \Gamma(\alpha+\beta-r)} t^{\alpha+\beta-r-1+\omega}+K t^{\alpha+\beta-r-1+\omega} g^{1 / q}(t)\right\} \\
& \leq \frac{c_{0}}{\Gamma(\beta)} t^{\beta-1}+\frac{c_{1} \Gamma(\alpha-r)}{\Gamma(\alpha) \Gamma(\alpha+\beta-r)} t^{\alpha+\beta-r-1} \\
& \quad+K t^{\alpha+\beta-r-1}\left[\left[(1-m) A(t)+B^{1-m}(t)\right]^{\frac{1}{1-m}}\right]^{1 / q} \\
& =\frac{c_{0}}{\Gamma(\beta)} t^{\beta-1}+\frac{c_{1} \Gamma(\alpha-r)}{\Gamma(\alpha) \Gamma(\alpha+\beta-r)} t^{\alpha+\beta-r-1+} \\
& \quad+K t^{\alpha+\beta-r-1}\left[(1-m) A(t)+B^{1-m}(t)\right]^{\frac{1}{q(1-m)}} .
\end{aligned}
$$


Case 2: $m=1$. In this case, for $t \in[0, T)$

$$
g(t) \leq B(t) e^{A(t)}
$$

Therefore,

$$
\begin{aligned}
& |u|=t^{-\omega} z(t) \\
& \leq \frac{c_{0}}{\Gamma(\beta)} t^{\beta-1}+\frac{c_{1} \Gamma(\alpha-r)}{\Gamma(\alpha) \Gamma(\alpha+\beta-r)} t^{\alpha+\beta-r-1}+K t^{\alpha+\beta-r-1} g^{1 / q}(t) \\
& \leq \frac{c_{0}}{\Gamma(\beta)} t^{\beta-1}+\frac{c_{1} \Gamma(\alpha-r)}{\Gamma(\alpha) \Gamma(\alpha+\beta-r)} t^{\alpha+\beta-r-1}+K t^{\alpha+\beta-r-1} B^{1 / q}(t) e^{A(t) / q} .
\end{aligned}
$$

This completes the proof.

\section{Acknowledgments:}

The author is grateful for the support provided by King Fahd University of Petroleum \& Minerals and the financial support by BAE Systems through the PDSR program by the British Council in Saudi Arabia. The research herein was performed in part while the author was visiting the Oxford Center for Collaborative Applied Mathematics (OCCAM), University of Oxford.

\section{References}

[1] A. A. Kilbas, H. M. Srivastava, and J. J. Trujillo, Theory and Applications of Fractional Differential Equations, vol. 204 of Mathematics Studies. Amsterdam: Elsevier, 2006 .

[2] I. Podlubny, Fractional Differential Equations, vol. 198 of Mathematics in Science and Engineering. Acad. Press, 1999.

[3] R. Hilfer, ed., Applications of Fractional Calculus in Physics, (Singapore), World Scientific, 2000.

[4] R. Caponetto, G. Dongola, L. Fortuna, and I. Petráš, Fractional Order Systems: Modeling and Control Applications, vol. 72 of World Scientific Series on Nonlinear Science. World Scientific, 2010.

[5] C. A. Monje, Y. Chen, B. M. Vinagre, D. Xue, and V. Feliu, Fractional-order Systems and Controls. Advances in Industrial Control, Springer, 2010.

[6] R. Hilfer, "Experimental evidence for fractional time evolution in glass forming materials," Chemical Physics, vol. 284, pp. 399-408, 2002.

[7] T. Wenchang, P. Wenxiao, and X. Mingyu, "A note on unsteady flows of a viscoelastic fluid with the fractional Maxwell model between two parallel plates," International Journal of Non-Linear Mechanics, vol. 38, pp. 645-650, 2003.

[8] F. Mainardi and R. Gorenflo, "Time-fractional derivatives in relaxation processes: A tutorial survey," Fractional Calculus \&6 Applied Analysis, vol. 10, no. 3, pp. 269-308, 2007. 
[9] F. Mainardi, Fractional Calculus and Waves in Linear Viscoelasticity. Imperial College Press, 2010.

[10] D. Baleanu, Z. B. Güvenç, and J. T. Machado, eds., New Trends in Nanotechnology and Fractional Calculus Applications, Springer, 2010.

[11] T. Sandev and Ž. Tomovski, "General time fractional wave equation for a vibrating string," Journal of Physics A: Mathematical and Theoretical, vol. 43, p. 055204, 2010.

[12] R. Klages, G. Radons, and I. Sokolov, eds., Anomalous Transport: Foundations and Applications, (Weinheim), Wiley-VCH, 2008.

[13] E. Gerolymatou, I. Vardoulakis, and R. Hilfer, "Modelling infiltration by means of a nonlinear fractional diffusion model," Journal of Physics D: Applied Physics, vol. 39, pp. 4104-4110, 2006.

[14] B. L. S. P. Rao, Statistical Inference for Fractional Diffusion Processes. Wiley, 2010.

[15] M. M. Meerschaert and A. Sikorskii, Stochastic Models for Fractional Calculus, vol. 43 of De Gruyter Studies in Mathematics. Berlin: De Gruyter, 2012.

[16] R. Metzler and J. Klafter, "The random walk's guide to anomalous diffusion: a fractional dynamics approach," Physics Reports, vol. 339, no. 1, pp. 1-77, 2000.

[17] R. Hilfer and L. Anton, "Fractional master equations and fractal time random walks," Physical Review E, vol. 51, pp. R848-R851, 1995.

[18] Y. Zhang, D. A. Benson, M. M. Meerschaert, E. M. LaBolle, and H. P. Scheffler, "Random walk approximation of fractional-order multiscaling anomalous diffusion," Physical Review E, vol. 74, pp. 026706-026715, 2006.

[19] B. Baeumer, M. M. Meerschaert, and E. Nane, "Brownian subordinators and fractional cauchy problems," Trans. Amer. Math. Soc., vol. 361, pp. 3915-3930, 2009.

[20] E. Scalas, R. Gorenflo, and F. Mainardi, "Fractional calculus and continuous-time finance," Physica A, vol. 284, pp. 376-384, 2000.

[21] E. Scalas, R. Gorenflo, F. Mainardi, and M. Meerschaert, "Speculative option valuation and the fractional diffusion equation," in Proceedings of the IFAC Workshop on Fractional Differentiation and its Applications, (FDA 04), Bordeaux, 2004. (J. Sabatier and J. T. Machado, eds.), 2004.

[22] D. Fulger, E. Scalas, and G. Germano, "Monte Carlo simulation of uncoupled continuous-time random walks yielding a stochastic solution of the space-time fractional diffusion equation." Physical Review E: Statistical, Nonlinear, and Soft Matter Physics, vol. 77, pp. .021122-021122, 2008.

[23] M. D. Ortigueira, Fractional Calculus for Scientists and Engineers, vol. 84 of Lecture Notes in Electrical Engineering. Springer, 2011.

[24] I. Petráš, Fractional-Order Nonlinear Systems: Modeling, Analysis and Simulation. Springer, 2011. 
[25] V. Lakshmikantham, S. Leela, and J. V. Devi, Theory of Fractional Dynamic Systems. Cambridge Scientific Publishers, 2009.

[26] K. Diethelm, The Analysis of Fractional Differential Equations. Springer, 2010.

[27] R. P. Agarwal, Y. Zhou, and Y. He, "Existence of fractional neutral functional differential equations," Computers and Mathematics with Applications, vol. 59, pp. 10951100, 2010.

[28] H. K. Avad and A. V. Glushak, "On perturbations of abstract fractional differential equations by nonlinear operators," Journal of Mathematical Sciences, vol. 170, no. 3, pp. 306-323, 2010.

[29] M. Kirane and S. A. Malik, "The profile of blowing-up solutions to a nonlinear system of fractional differential equations," Nonlinear Analysis, vol. 73, pp. 3723-3736, 2010.

[30] G. Chai, "Existence results for boundary value problems of nonlinear fractional differential equations," Computers and Mathematics with Applications, vol. 62, pp. 2374$2382,2011$.

[31] S. Zhang and X. Su, "The existence of a solution for a fractional differential equation with nonlinear boundary conditions considered using upper and lower solutions in reverse order," Computers and Mathematics with Applications, vol. 62, pp. 12691274, 2011.

[32] A. Babakhani and D. Baleanu, "Employing of some basic theory for class of fractional differential equations," Advances in Difference Equations, vol. 2011, pp. 1-13, 2011.

[33] K. M. Furati, M. D. Kassim, and N. e. Tatar, "Existence and uniqueness for a problem involving Hilfer fractional derivative," Computers and Mathematics with Applications, 2012. In press.

[34] K. M. Furati, "A Cauchy-type problem with a sequential fractional derivative in the space of continuous functions," Boundary Value Problems, vol. 2012:58, p. 14 pages, 2012. doi:10.1186/1687-2770-2012-58.

[35] A. V. Glushak, "Cauchy-type problem for an abstract differential equation with fractional derivatives," Mathematical Notes, vol. 77, no. 1, pp. 26-38, 2005. Translated from Matematicheskie Zametki, vol. 77, no. 1, 2005, pp. 28-41.

[36] A. V. Glushak, "On the properties of a Cauchy-type problem for an abstract differential equation with fractional derivatives," Mathematical Notes, vol. 82, no. 5, pp. 596-607, 2007. Translated from Matematicheskie Zametki, 2007, Vol. 82, No. 5, pp. $665-677$.

[37] A. V. Glushak, "Correctness of Cauchy-type problems for abstract differential equations with fractional derivatives," Russian Mathematics, vol. 53, no. 9, pp. 0-19, 2009. Translated from Izvestiya Vysshikh Uchebnykh Zavedenii. Matematika, 2009, No. 9, pp. 13-24.

[38] K. M. Furati, "A cauchy-type problem involving a weighted sequential derivative," in In the 5th IFAC Symposium on Fractional Differentiation and its Applications (FDA12). Nanjing, China, 2012. 
[39] B. G. Pachpatte, Inequalities for Differential and Integral Equations, vol. 197 of Mathematics in Science and Engineering. Acad. Press, 1998.

[40] M. W. Michalski, Derivatives of Noninteger Order and their Applications. PhD thesis, Polska Akademia Nauk, 1993.

[41] S. G. Samko, A. A. Kilbas, and O. I. Marichev, Fractional Integrals and Derivatives, Theory and Applications. Amsterdam: Gordon and Breach, 1993. Engl. Trans. from the Russian 1987.

[42] A. C. McBride, Fractional calculus and integral transforms of generalized functions. Pitman, 1979. 



\section{RECENT REPORTS}

12/50 Fourier spectral methods for fractional-in-space reaction-diffusion equations

Bueno-Orovio

Kay

Burrage

12/51 Meniscal tear film fluid dynamics near Marx's line

Zubkov

Breward

Gaffney

12/52 Validity of the Cauchy-Born rule applied to discrete cellular-scale models of biological tissues

Davit

Osborne

Byrne

Gavaghan

Pitt-Francis

12/53 A thin rivulet or ridge subject to a uniform transverse shear stress at its free surface due to an external airflow

Sullivan

Paterson

Wilson

Duffy

12/54 The Stokes boundary layer for a thixotropic or antithixotropic fluid

McArdle

Pritchard

Wilson

12/55 Thermoviscous Coating and Rimming Flow

Leslie

Wilson

Duffy

12/56 On the anomalous dynamics of capillary rise in porous media

Shikhmurzaev Sprittles

12/57 Compactly supported radial basis functions: how and why? Zhu

12/58 Multiscale reaction-diffusion algorithms: pde-assisted Brownian dynamics

Franz

Flegg

Chapman

Erban

12/59 Numerical simulation of shear and the Poynting effects by the fi-

Mihai nite element method: An application of the generalised empirical inequalities in non-linear elasticity

Goriely

12/60 From Brownian dynamics to Markov chain: an ion channel example

Chen

Erban

Chapman

12/61 Three-dimensional coating and rimming flow: a ring of fluid on a rotating horizontal cylinder

Leslie

Wilson

Duffy

12/62 A two-pressure model for slightly compressible single phase flow in bi-structured porous media

Soulaine

Davit

Quintard

12/63 Mathematical modelling plant signalling networks

Muraro

Byrne

King

Bennett 
12/65 Effective order strong stability preserving RungeKutta methods

Hadjimichael

Macdonald

Ketcheson

Verner

12/66 Morphoelastic Rods Part I: A Single Growing Elastic Rod

Moulton

Lessinnes

Goriely

12/67 Wrinkling in the deflation of elastic bubbles

Aumaitre

Knoche

Cicuta

Vella

12/68 Indentation of ellipsoidal and cylindrical elastic shells

Vella

Ajdari

Vaziri

Boudaoud

12/69 Memory of Recessions

Cross

McNamara

Pokrovskiif

12/70 An estimate of energy dissipation due to soil-moisture hysteresis

McNamara

12/71 The Mathematics Behind Sherlock Holmes: A Game of Shadows

Goriely

Moulton

12/72 Some observations on weighted GMRES

Güttel

Pestana

Copies of these, and any other OCCAM reports can be obtained from:

Oxford Centre for Collaborative Applied Mathematics

Mathematical Institute

24 - 29 St Giles'

Oxford

OX1 3LB

England

www.maths.ox.ac.uk/occam 